\title{
Computational Fluid Dynamics (CFD) for Predicting and Enhancing Intake Flow Problems of Pumping Stations
}

\author{
Sami Abdel Fattah, Ibrahim R. Teaima, and M. A. Hashem \\ Mechanical \&Electrical Research Institute, \\ National Water Research Center, Ministry of Water Resources \&Irrigation, \\ Delta Barrage, Egypt
}

\begin{abstract}
The disturbance in velocity distribution generates hydraulic instability of pumping units, which leads to failure, damage and other operation and maintenance difficulties. The objective of this research is to predict and enhance the hydraulic problems in the sump intake of El-Tabya pumping station. The station consists of six axial pumping large units with discharge of $7 \mathrm{~m}^{3} / \mathrm{sec}$ for each unit, 5.5 static head. Three-dimensional Computational Fluid Dynamics (CFD) model is used to simulate the flow conditions at different working pumping units and different water levels to predict the hydraulic problem at the suction side. In this study, five different cases were studied at existing operating conditions and when add routers in suction sump. The research show that existing sump shape generates swirl flow and appear dead zones in the inner curvature of the intake when operate six units at the same time. The installation of routers with curvature length $6 \mathrm{~m}$ and width $0.5 \mathrm{~m}$ for each pump reduce the pre-swirling surface flow and improves the suction flow condition.
\end{abstract}

Keywords: Hydraulic stability; Pre-swirling; CFD; Velocity distribution; Sump intake

\section{INTRODUCTION:}

It is an accepted fact that faulty design of pump sump or intake is one of the major causes of unsatisfactory operation of pumps in any pumping station. The efficiency and performance of pumping stations involving multiple pumping units depend not only on the efficiency of the pumping units but also on the proper design of the intake sump. El-Tabya Pumping Station located at El-Tabya drainage in Alexandria governorate was established in 2001 to remove drainage water from 45000 fedans, it consists of six axial pumping large units with discharge of $7 \mathrm{~m}^{3} / \mathrm{sec}$ for each unit, 5.5 static head. The pumps driven by $650 \mathrm{kw}$ electric motor with gearbox. Figure (1) shows the location of the station. The operating system depends on running not more than four pumping units.

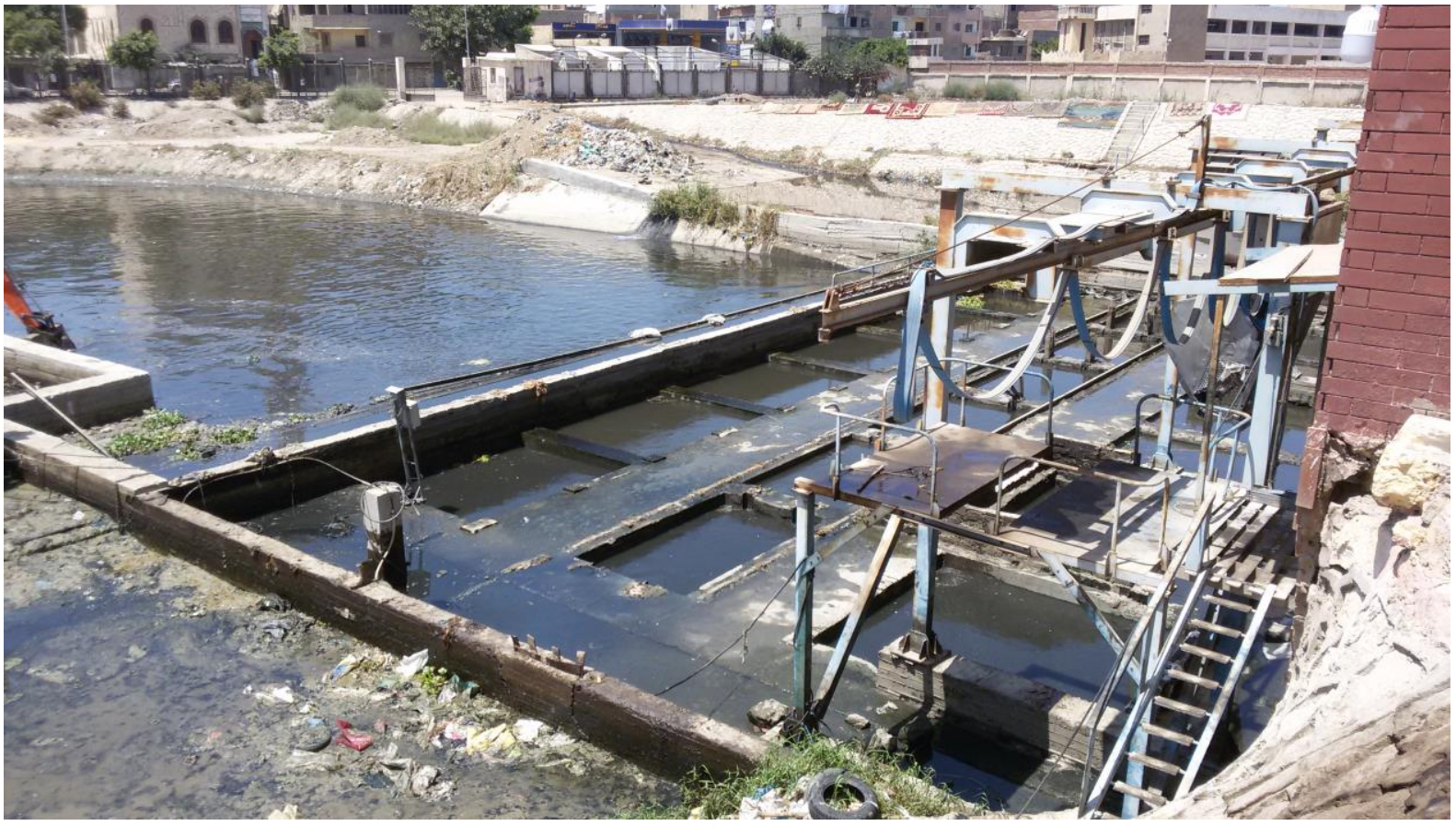

Figure 1: Intake of El-Tabya pumping station

To design such compact and high-speed pump sumps, it is necessary to avoid the generation of strong vortices that induce harmful vibration and noise. According to the JSME criteria for a pump sump design (Standard Method for Model Testing the 
Performance of a Pump Sump 1984), a submerged vortex with a vapor core should preclude. Because this type of vortex leads to reduce the pump efficiency, induce vibrations and excessive bearing loads, it results in operational and maintenance cost, leading to other operating difficulties. So far, experiments on scale models have been performed to assess sump performance and to develop a suitable sump design. These experiments, however, are costly and time-consuming. Hence, alternative evaluation methods ware needed. Kamemoto et al. (1986) presented numerical results of basic flow features in a pump sump by using a three-dimensional discrete vortex method. Takata et al. (1992) reproduced a strong cavitating vortex in a pump sump by the Large Eddy Simulation. Codes that are more practical based on the Reynolds-Averaged Navier-Stokes equations were also applied to flow in pump sumps, and it was shown that general flow patterns, which induce the submerged vortices, agreed well with experiments (Constantinescu \& Patel 1998; Enomoto et al. 1995). The common solution for avoiding air entrainment and swirl is to provide sufficient submergence to the intake. If the required approach flow conditions cannot be met to avoid swirl and air entrainment, it is economical to consider other anti-vortex devices for preventing vortices at water intakes. The antivortex device reduces the operation and maintenance cost of pumping units exposed to vortex problems. However, the effect of extreme pressure variation in a pressurized flow due to air entrainment is shown by Kabiri-Samani et al. (2006). There are several means of avoiding air entrainment where, for large structures, the most cost-effective option is often determined by a physical model study. One of the most popular ways is the use of special vortex suppression devices (Knauss 1987). Ample research work has been carried out to have a better knowledge of vortex formation and critical submergence depths for vertical pipe intakes. Among the analytical approaches, the works of Odgaard (1986), Hite, and Mih (1994) are more relevant. However, for experimental published work, the studies can be divided into two categories. First, the understanding of free vortex hydrodynamics (Anwar et al. 1978; Carriveau 2006; Daggett \& Keulegan 1974; Jain et al. 1978; Padmanabhan \& Hecker 1984; Rocklage-Marliani et al. 2003) and second, parameters affecting the critical submergence depth (Anwar et al. 1978; Borghei \&Kabiri Samani 2003; Reddy \& Pickford 1972; Yildirim 2004; Yildirim \& Kocabas 1995, 2002). On the other hand, many attempts have been made numerically to analyze the hydrodynamics of vortex and cavity development (Emdad \& Nikseresht 2003; Hannani \& Parsinejad 2003). The objective of this research is to study the modifications of existing sump intake of El Tabya pumping station. This change in the design of the suction sump and suction channel is an improving suction flow condition. Moreover, attempt to eliminate the mechanical and hydraulic problems, leads to reduce the cost of operation and maintenance costs.

\section{NUMERICAL ANALYSIS}

From a purely numerical perspective, the geometric complexity of the problem is such that it demands the full power of modern computational fluid dynamics (CFD) to solve the equations of motion and turbulence models in domains that involve multiple surfaces, such as sump floor surface, bell mouth and sump free surface. Additional difficulties are associated with modeling free surface and vortex phenomenon, the physical characteristics of which are not yet fully understood. Free surface vortex type class (2) according to American National Standard Institute (Hydraulic Institute 1998) was studied numerically. The free surface and subsurface vortices entering the pump must be less severe than vortices with diffusive dye cores. Free surface vortices at intakes can cause excessive vibration, efficiency loss, structural damage, and flow reduction in hydroturbines, pumps, culverts, and so on. They can also be a safety hazard involving a potential loss of life. In spite of the practical importance of the problem, the literature on numerical modeling of pump intake flows is rather limited.

In the present work, an attempt has been made to simulate and predict the flow conditions such as vortices and swirl for different operating conditions, with an aim to determine the flow problems of El Tabya pumping station.

\subsection{Building a CFD model}

This model solves the Reynolds-averaged Navier-Stokes (RANS) equations for steady, incompressible turbulent flows in a pump intake. The mass and momentum equations are written, in Cartesian tensor form, as

$$
\begin{aligned}
& \frac{\partial\left(\rho U_{j}\right)}{\partial x_{j}}=0 \\
& \frac{\partial\left(\rho U_{i}\right)}{\partial t}+\frac{\partial\left(\rho U_{i} U_{j}\right)}{\partial x_{j}}=-\frac{\partial P}{\partial x_{i}}+\frac{\partial}{\partial x_{j}}\left(\mu \frac{\partial U_{i}}{\partial x_{j}}-\rho \overline{u_{i} u_{j}}\right)
\end{aligned}
$$

where $U_{j}$ and $u_{j}$ are jth components of the mean and fluctuating velocities, $P$ is the piezometric pressure and $\rho$ and $\mu$ are fluid density and viscosity, respectively.

The Reynolds stresses $\rho u_{i} u_{j}$ are expressed in terms of the mean rate of strain using Boussinesq's eddy-viscosity formula:

$$
\rho \overline{u_{i} u_{j}}=\mu_{t}\left(\frac{\partial U_{i}}{\partial x_{j}}+\frac{\partial U_{j}}{\partial U_{i}}\right)-\frac{2}{3} \rho k \delta_{i j}
$$

where $\mu_{\mathrm{t}}=$ turbulence viscosity, $\delta_{\mathrm{ij}}=$ the Kronecker symbol and $\mathrm{k}=$ turbulence kinetic energy per unit mass. For the present applications, the standard $\mathrm{k}-\varepsilon$ model [26] is used. The eddy viscosity is obtained from 
$\mu_{t}=C_{\mu} \rho \frac{k^{2}}{\varepsilon}$

where $\varepsilon=$ turbulence dissipation rate. The transport equations for $\mathrm{k}$ and $\varepsilon$ are expressed as

$$
\begin{aligned}
& \frac{\partial\left(\rho U_{j} k\right)}{\partial x_{j}}=\frac{\partial}{\partial x_{j}}\left[\left(\mu+\frac{\mu_{t}}{\sigma_{k}}\right) \frac{\partial k}{\partial x_{j}}\right]+G-\rho \varepsilon \\
& \frac{\partial\left(\rho U_{j} \varepsilon\right)}{\partial x_{j}}=\frac{\partial}{\partial x_{j}}\left[\left(\mu+\frac{\mu_{t}}{\sigma_{\varepsilon}}\right) \frac{\partial \varepsilon}{\partial x_{j}}\right]+C_{\varepsilon 1} \frac{\varepsilon}{k} G-C_{\varepsilon 2} \rho \frac{\varepsilon^{2}}{k}
\end{aligned}
$$

Where $G=-\rho \overline{u_{i} u_{j}}\left(\frac{\partial U_{i}}{\partial x_{j}}\right)$ is the generation of k. The turbulence model coefficients are: $\mathrm{C}_{\mu}=0.09, \sigma_{\mathrm{k}}=1.0, \sigma_{\varepsilon}=1.3, \mathrm{C}_{\varepsilon 1}=1.44$ and $\mathrm{C}_{\varepsilon 2}=1.92$.

The finite volume approach was employed for the discretization of the governing equations. The discretization in space is of second order accuracy. The physical domain was meshed into a number of cells in which all dependent variables are stored at the geometric centers of each volume cell. The equations are integrated over cells using the Gauss theorem.

\subsection{Numerical simulation of the intake model}

Several scenarios for the operation of stopping units and operating other units to reach the best scenarios for the operation of units within the station, to identify and analyze the problem to select the best scenarios to control flow within the stream and entrances units. Figure (2) shows a two-dimensional overall pump intake configuration of the pump intake model simulated in this study. Figure (3) shows a three dimensional image of the suction sump respectively. The three-dimensional unstructured mesh (tetrahedral) has been created by using the structured mesh (hexagonal).

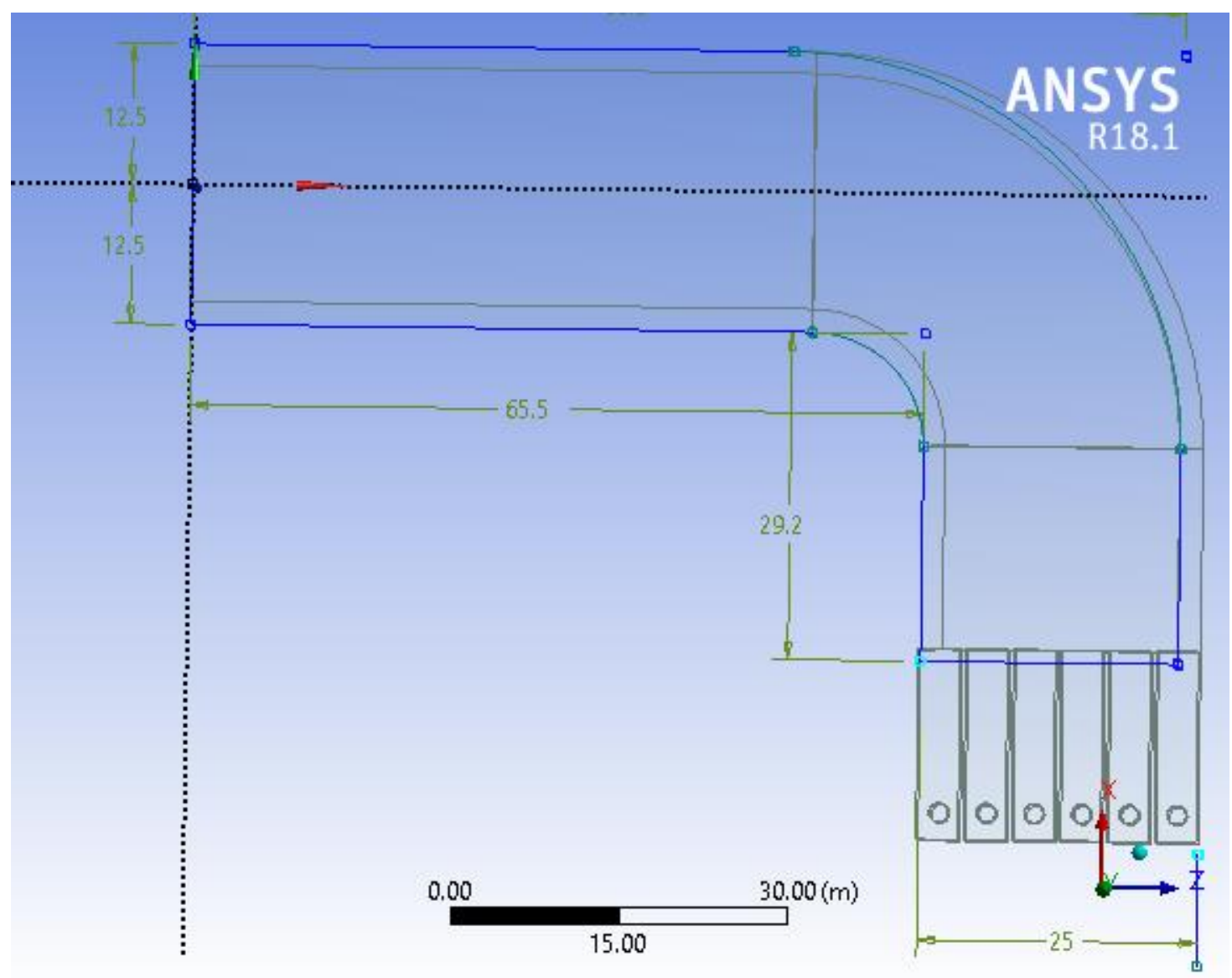

Figure 2: Two-dimensional image of the suction sump and unit input. 


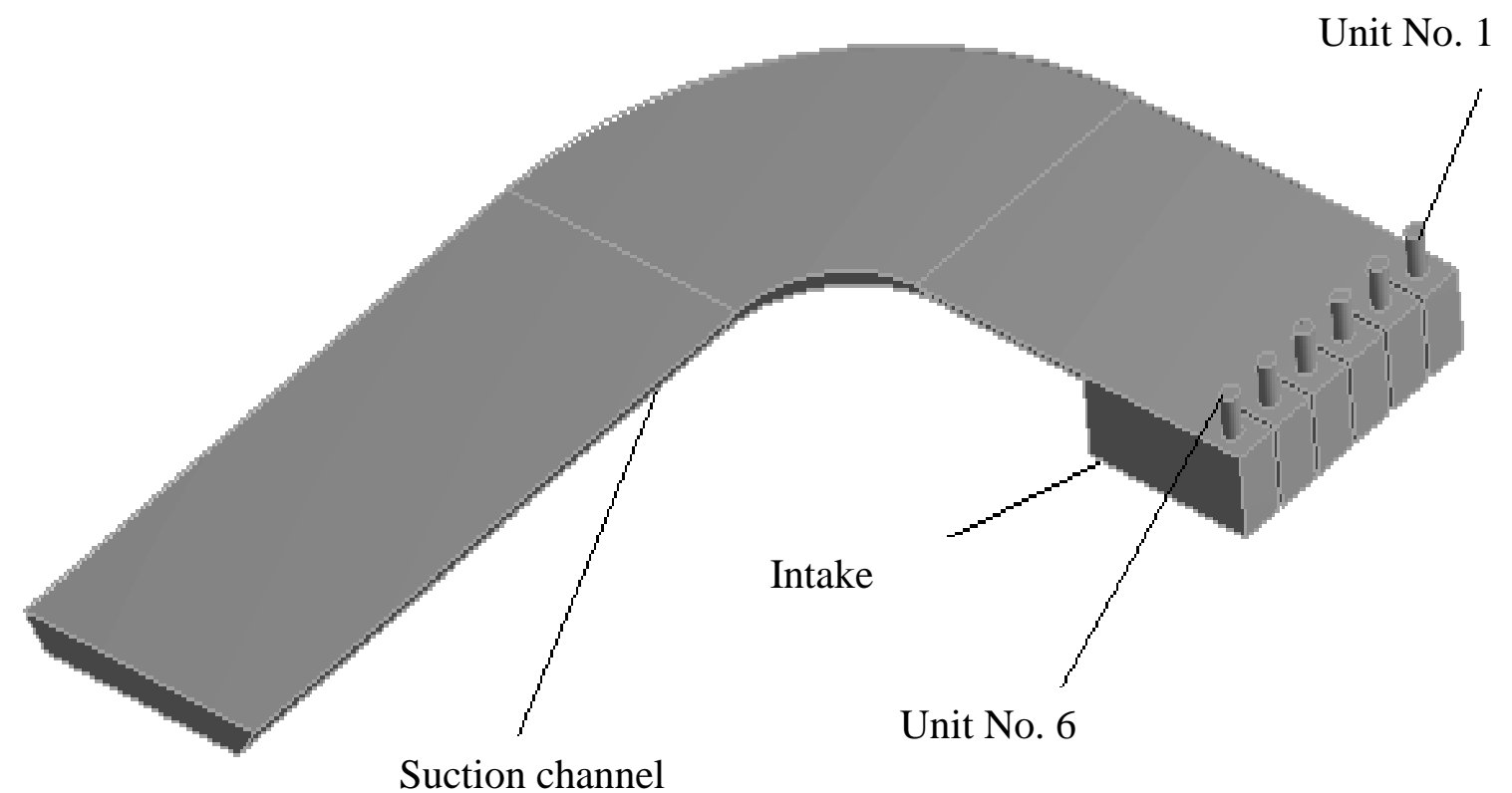

Figure 3: Three-dimensional image of the suction basin and entrance of the units

\subsection{Boundary Conditions and Grid Generation}

To get an accurate simulation, the outlet volume flow applied in these two cases. The numerical analysis is transient state. The "ideal wall" condition simulates the level of water, while the environment pressure condition is applied to simulate on the "inlet caps". The quality of the computational mesh has an important role in achieving the desired accuracy of the simulations especially if the computational domain is very complex. The basic three-dimensional geometry is prepared using the ANSYS R18.1 software flow simulation software drawing with the grid surfaces plot of sump. The equations are supplemented by fluid state equations defining the nature of the fluid, and by empirical dependencies of fluid density, viscosity and thermal conductivity on temperature. To predict turbulent flows, the Favre-averaged Navier-Stokes equations are used, where timeaveraged effects of the flow turbulence on the flow parameters are considered, where the other, i.e. large-scale, time-dependent phenomena are taken into account directly. Three dimensional unstructured meshes are used for the flow simulation in the pump sump. Unstructured mesh is used for this study due to model complexity and easy to be mesh especially at the intake section. The numerical solver uses unstructured meshes which allow flexibility in meshing very complex geometries while maintaining high quality computational mesh which is necessary for obtaining accurate solutions (B. E. Launder and D. B. Spalding, 1974).

\section{RESULTS \& DISCUSSIONS FOR ORIGINAL SUMP MODEL}

The existing operating condition contains five scenarios; these Scenarios were done to describe the shapes of the flow, its lines and directions, as well as forms of speeds for each five scenarios to see the best operating conditions for the units as follows. Then the same five scenarios were done but after placement routers (walls) close to the bend of the suction channel to redistribute the lines flow within the stream at the entrance of units.

\subsection{First case (all six units operating)}

For the first scenario, when all six units' ware operated, it is found that through the width of the flow lines to the maximum level of water, the free surface. The flow lines are dense with the external bend of the suction path beam (through units 1,2 and 3 ), while it decreases with the internal bend of the suction path (through units 4, 5 and 6), as shown in figure (4-a). On the other side figure (4-a) shows the at level of the bell, streamlines irregularity distribution of the internal flow lines of the six units and are dense in the direction of the outer curvature of the suction sump and less of the external bend, as shown in figure (4-b). It is also the velocity distribution of the suction channel and the intake shows a relatively a static area (dead) with relatively low speeds towards unit number 6, as shown in figure (4-b). In another hand, it has found that from the shape of flow directions at the bottom level of the suction pipes a circulation separation zones appeared at the sump intake under the suction pipe and above the sump floor. 


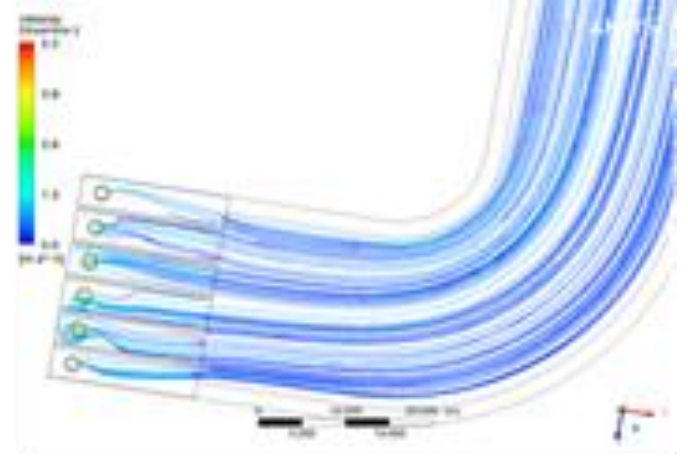

(a) Distribution of the internal flow lines of the six units

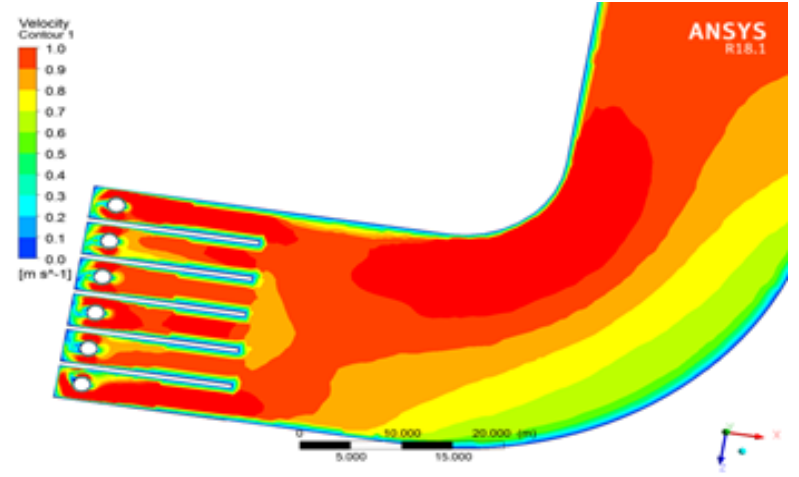

(b) Velocity distribution of the channel and the entrance

Figure 4: First case, all six units operating

\subsection{Second case (three units operating $(1,2 \& 3)$}

From the results of the first scenario, it's clear that there is a difference in the distribution of the flow lines between the units located on the external and internal bending side of the sump. Therefore, we suggested doing another scenario by running the units separately to observe the flow distribution. In the second scenario, when three units are operated (units 1,2 and 3 which located in the outer bend of the suction path), it is found that the behavior of the stream lines is more uniform in the outer bend of the suction path, than that of the internal bend. Swirl were appeared at the entrance of units $(4,5,6)$, as shown in figure $(5-a)$ which shows the flow lines in the suction basin and the intake of the pumps. Also at the level of the bell it is found that the direction of the flow lines is regular distribution for units $(1,2,3)$, and the swirls were appeared at the units $(5,6)$, as shown in figure (5-a) which shows the flow lines for different units in water level. However, the velocity distribution shows that there is a drop zone of the velocity values on the inner bend of the intake, as shown in figure (5-b). While from the flow directions at the level of the bell it's appears that presence of places of separation and swirls at unit 1 .

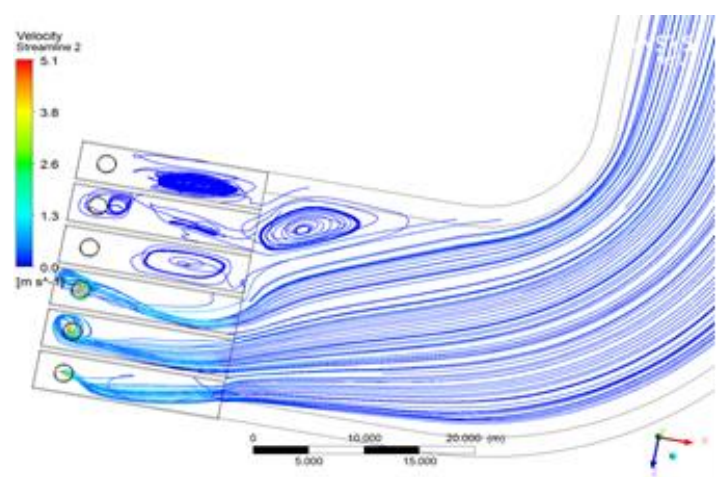

(a) Flow lines for different units at the level below the bell level.

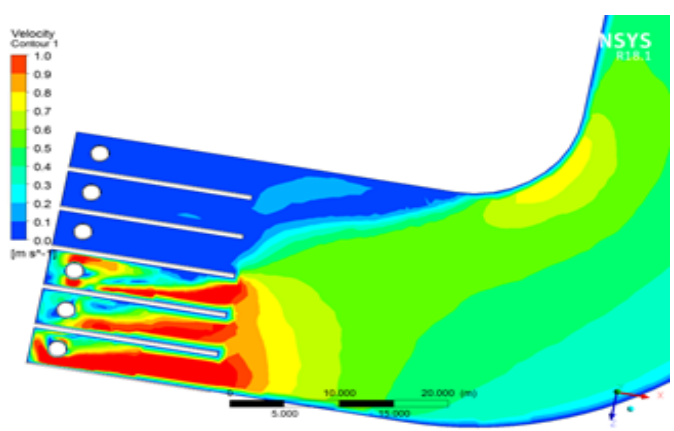

(b) Velocity distribution of the channel and the entrance

Figure 5: Second case (three units operating $(1,2 \& 3)$

\subsection{Third case (three units operating (4 \&5\&6)}

In the third scenario when three units are operated (units 4,5 and 6 which located in the inner bend of the suction intake), it is found that irregularity the lines of flow at the maximum level, opposite direction for lines of flow far from the units $(6,5$ and 4$)$, effects of the sharp curvature of the stream in the form of flow lines where it's disappear at units $(4,6)$, as shown in figures $(6-$ a). While, at the level of the bell the it's clear the effect of turbulent flow by sudden change in the direction of the flow lines before the intake of the working units and vortices in unit 6, as shown in figure (6-a). However, a map of velocities shows irregularities of the speeds at the intake of the three units, as shown in figure (6-b). Finally, from the flow directions at the level of the bell it's appears presence of separation zones and vortices at the bell level for the three units. 


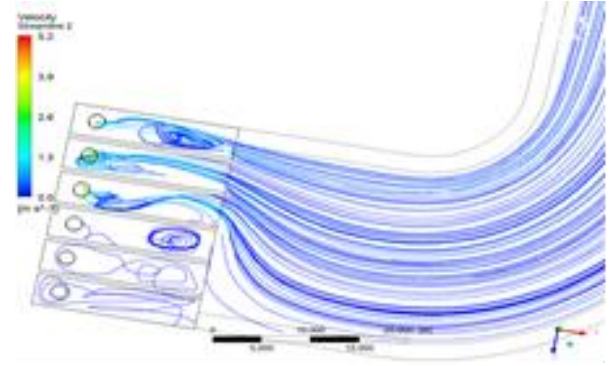

(a) Flow lines at the bell level.

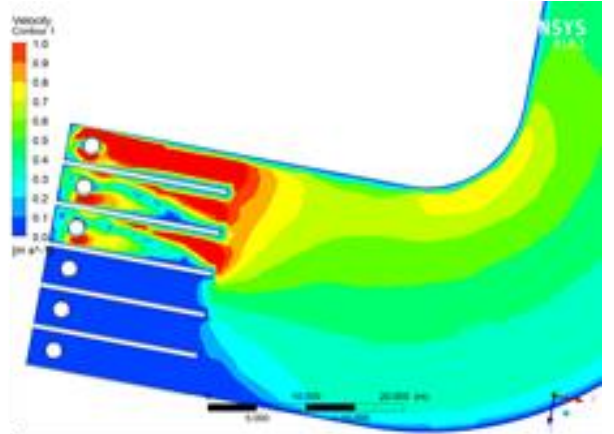

(b) Velocity distribution of the channel and the entrance

Figure 6: Third case (three units operating $(4,5 \& 6)$

\subsection{Forth case (four units operating $(1,3,4 \& 6)$}

In the fourth scenario when four units are operated (units 1,3,4 and 6), it is found that the flow lines were not distributed regularly on the bending and at the entrance of suction units, decreasing intensity of the flow lines in suction units, as shown in figure (7-a). In addition, streamlines of the bell level indicate irregularity of the incoming lines of the units, as shown in figure (7-a). However, a velocity distribution of streamlines shows that still irregular flow velocities within the entrances of the working units, as shown in figure (7-b). While from the flow directions it's appears that there are areas of separation of the flow and the effect of the whirls at the level of the bell, especially for unit's $(1,6)$.

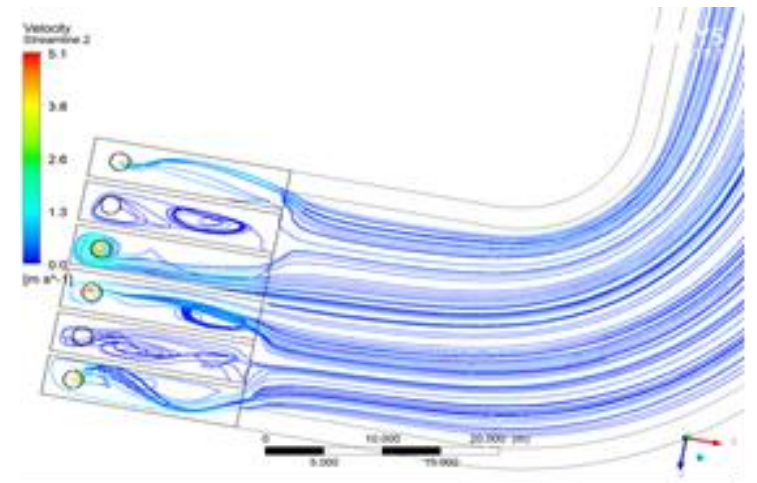

(a) Flow lines at the bell level for the fourth scenario.

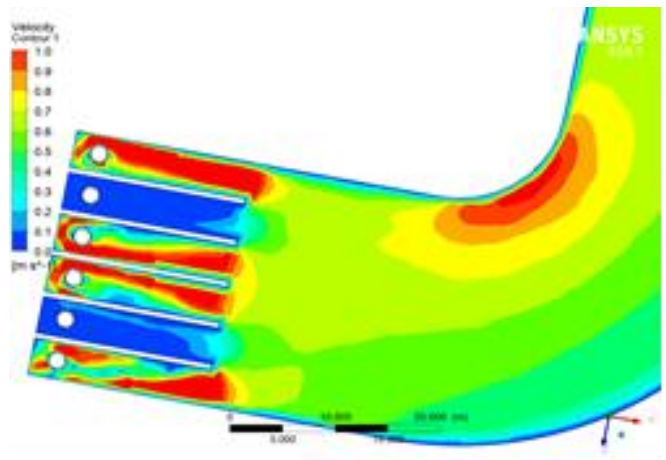

(b) Velocity distribution of the channel and the entrance

Figure 7: Forth case (four units operating $(1,3,4 \& 6)$

\subsection{Fifth case (four units operating $(2,3,4 \& 5)$}

In the fifth scenario when four units are operated (units 2,3,4 and 5), it is found that the flow lines indicate regularity in the distribution of lines of flow on both sides of the suction of units, as shown in figure (8-a). Also at the level of the bell it is found that organization of the water flow lines, as shown in figure (8-a). However, the velocity distribution was affected by the internal bending of the intake at relatively low velocity values, as shown in figure (8-b). While from the flow directions at the level of the bell it's illustrated that the units are affected by the presence of break-down zones under the bell.

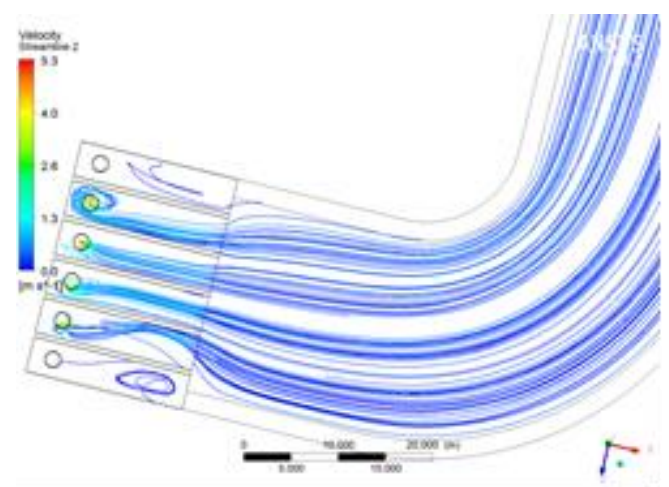

(a) Flow lines at the bell level for the fifth scenario.

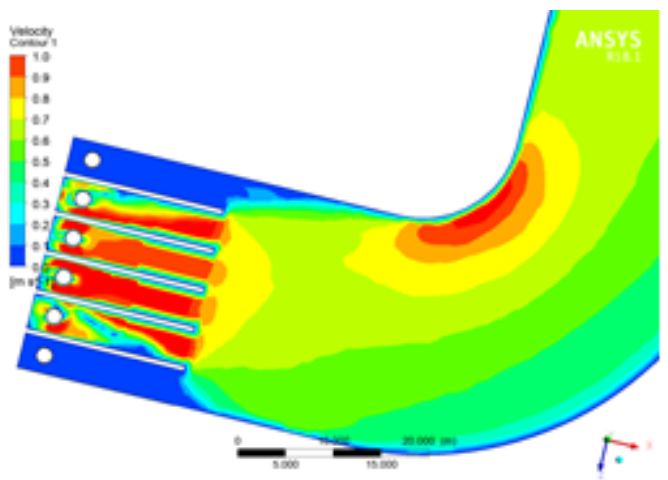

(b) Velocity distribution of the channel and the entrance

Figure 8: Fifth case (four units operating $(2,3,4 \& 5)$ 
From the results above there are two problems were occurred, first problem is the poor distribution for the streamlines of the water at the suction to the units due to a sharp bend in the suction sump that impedes the stability of flow in the entrance of the units, especially when units $(5,6)$ or $(1,2)$ run together at the same time. Where the second problem is the presence of areas where velocities are relatively low so, it become places of accumulation of sediments, which affects the work of the units.

Therefore, a redistribution of the flow lines in the suction basin and in the entrance of the units was suggested to solve these problems. First solutions were the placement of routers or walls close to the bend of the suction channel at the end of the bend. These routers are 23.5 meters from the entrance to the station. Sequentially these routers are corresponding to the wall separating the units, to regulate and redistribute the streamlines at the entrance of units. The length of every routers is six meters and 0.5 meters' width and be located as shown in figure (9). The second solutions were width the suction channel from the inner side. This modifications are responsible for disappear of pre-swirl and vortex flow.

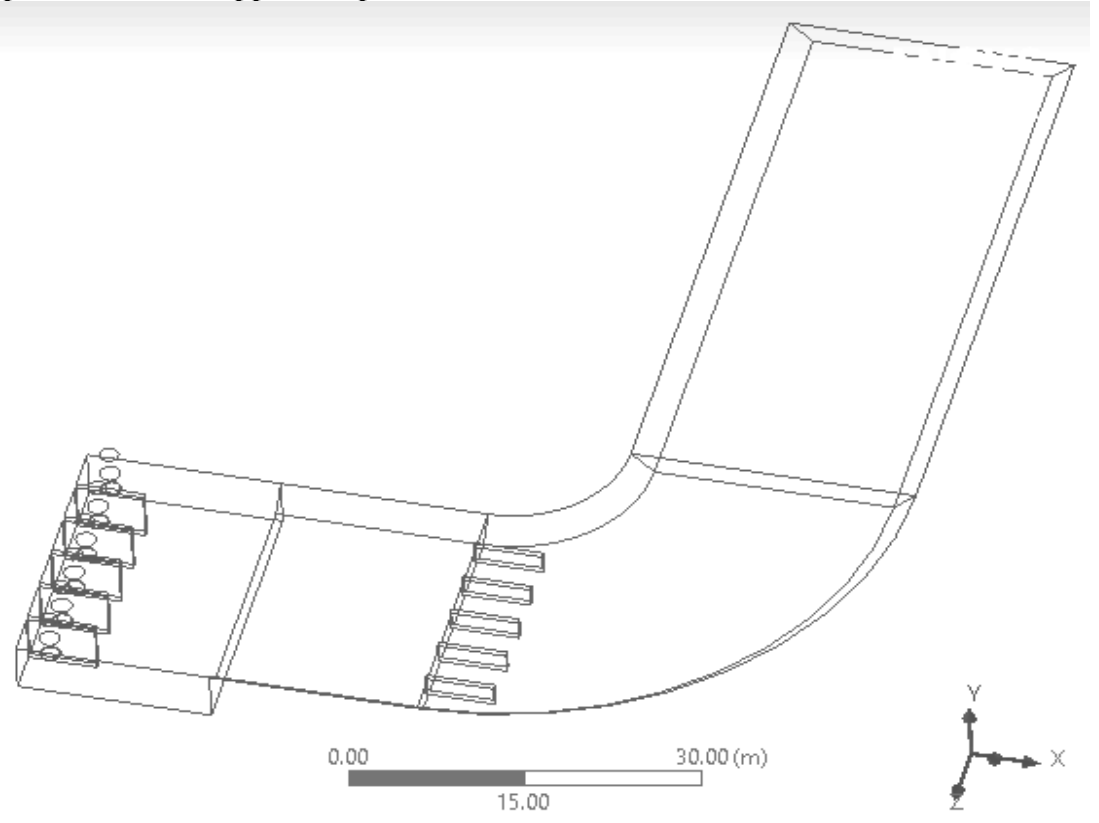

Figure 9: Setting the proposed routers through the suction sump

\section{MODIFICATION OF THE TESTED SUMP BY ADDING ROUTER}

Following the original suction sump design testing, a series of design modification tests were conducted. The objective of these tests is to develop and implement changes to the existing suction sump that would reduce or eliminate the adverse flow conditions observed for the original sump geometry.

\subsection{First case (all six units operating)}

Figure (10) illustrates the installation of routers are 23.5 meters from the entrance to the station. The results of the flow lines of the first scenario after adding router show good redistribution of the flow lines at the entrance of the units, especially at units ( 5 \& 6) which had the flow lines, as shown in Figure (10-a). In addition, it is appearing that a good distribution of the internal flow lines for all six units working, especially the units that were previously defective, namely the units $(5,6)$, as shown in Figure (10-b). And also, the speed map shows velocity become uniform in the intake and at the entrance unit, as shown in Figure (10-c). Finally, the flow directions at the level of the bell show decreasing density separation places and vortex locations below the units, as shown in Figure (10-d).

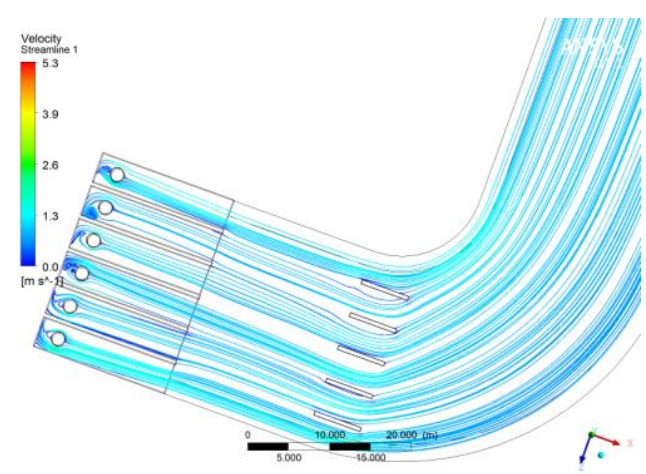

(a) Flow lines at a maximum level after adding router

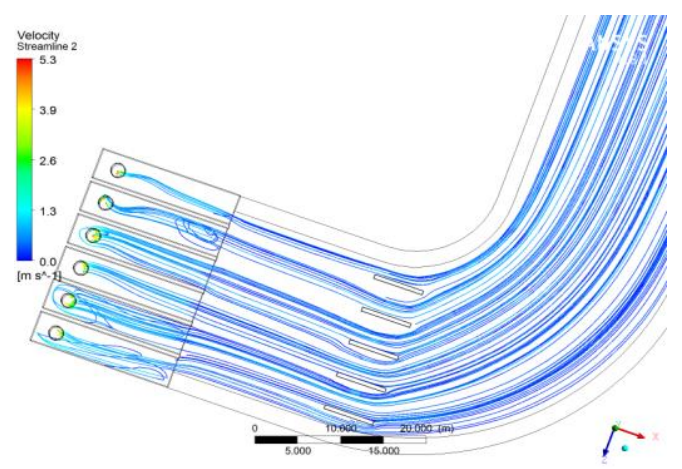

(b) Flow lines at the level of the bell after adding router 


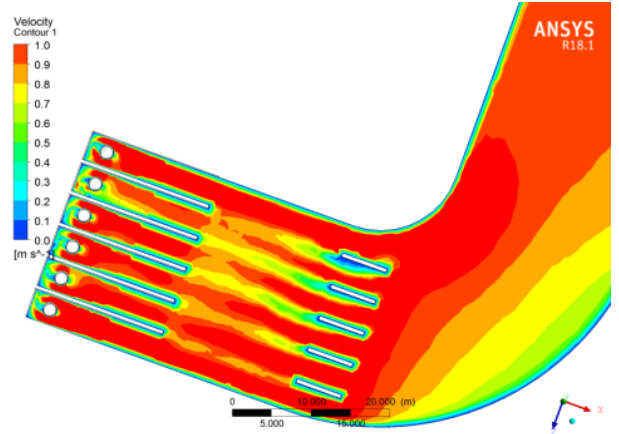

(c) Map of velocities after adding routers

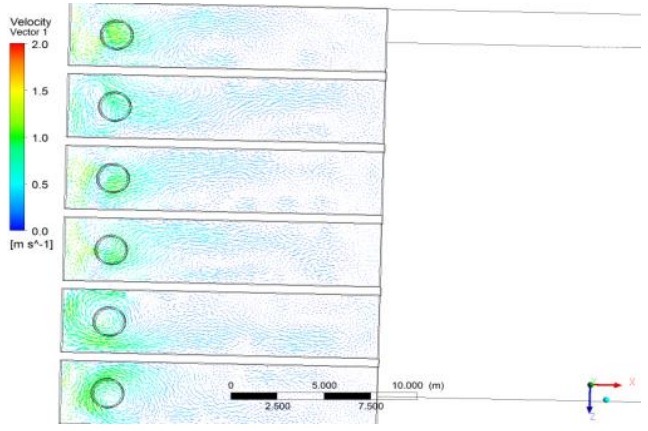

(d) Flow directions at the bell level after adding routers

Figure 10: First case: all six units operating

\subsection{Second case (three units operating $(1,2 \& 3)$}

The results of the flow lines of the second scenario after adding routers indicate disappearance of the vortices the maximum level on the units $(4,5,6)$, as shown in Figure (11-a) and disappearance of swirls at the level of the bell on the units $(4,5,6)$, as shown in Figure (11-a). Also, it is clear that the routers worked on the uniformity of the velocity values to the three working units but with relatively short area of the relatively low speeds of the inner bend of the tube, but improved better than in the case of the same case without routers, as shown in Figure (11-b). Finally, the flow directions at the level of the bell show regularity is observed for units (3.2) but there is breakout area below unit 1.

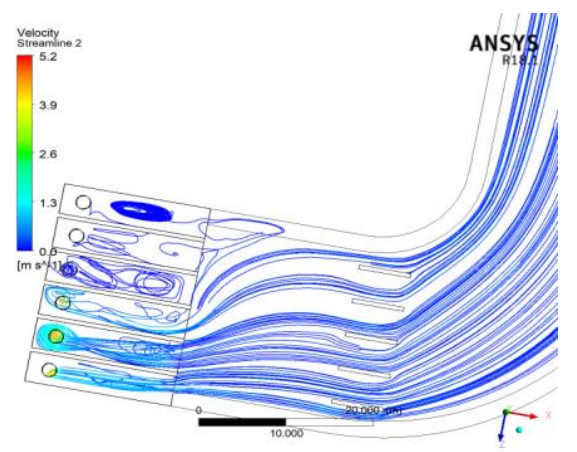

(a) Flow lines at the level of the bell for the second scenario after adding routers

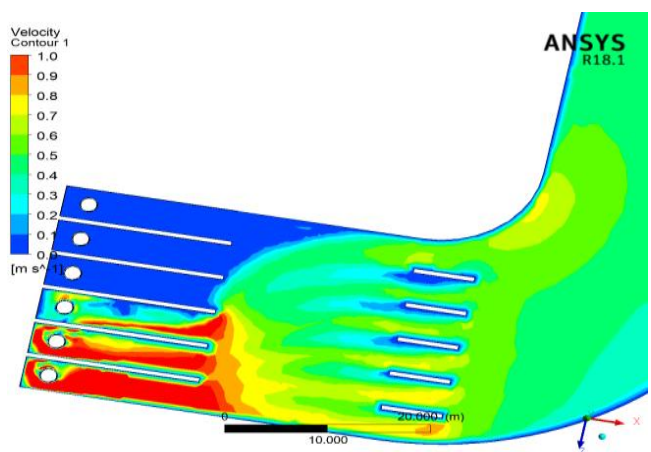

(b) Map of velocities for the second scenario after adding routers

Figure 11: Second case (three units operating $(1 \& 2 \& 3))$

\subsection{Third case (three units operating $(4,5 \& 6)$}

The results of the flow lines of the third scenario after adding routers indicate good regularity of the flow lines at maximum water level, as shown in Figure (12-a) and uniformity of pulling for units below the bell, as shown in Figure (12-a). In addition, the velocity values of the units are irregular as for the velocity map, as shown in Figure (12-b). As for the flow directions below the bell, it is appearing that the low effect of the swirl zones.

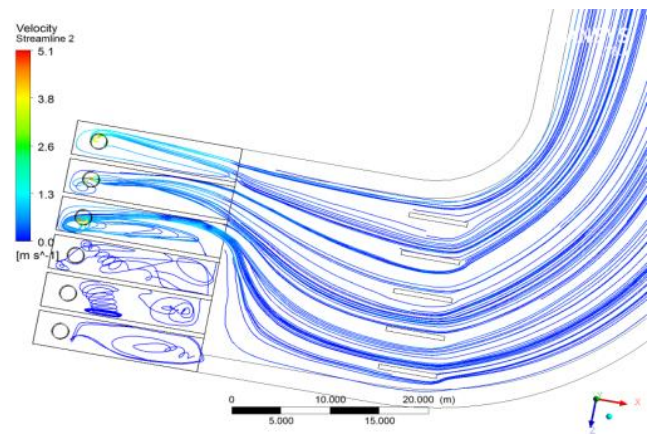

(a) Flow lines at the level of the bell for the third scenario after adding routers

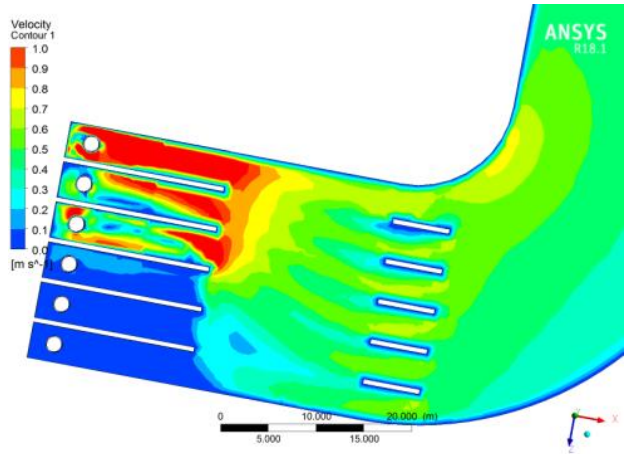

(b) Map of velocities for the third scenario after adding routers

Figure 12: Third case (three units operating $(4,5 \& 6)$ ) 


\subsection{Forth case (four units operating $(1,3,4 \& 6)$}

The results of the streamlines in case of forth scenario after adding routers indicates that, the streamlines become working uniformity as shown in Figure (13-a) and also it is appearing that a good distribution of the lines on all the units, as shown in Figure (13-a) while, figure (13-b) shows a good distribution of velocity for working units and finally, showing that the units (3, 4) aren't affected by the areas of the dissociation of the flow, while unit $(1,6)$ are effected.

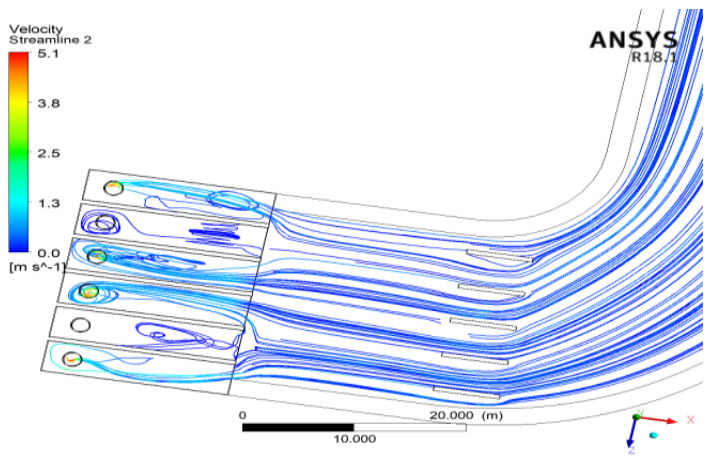

(a) Flow lines at the level of the bell for the forth scenario after adding routers

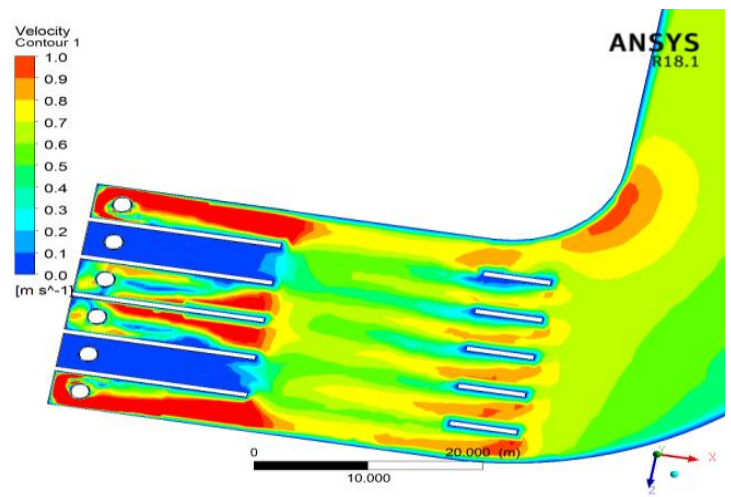

(b) Map of velocities for the forth scenario after adding routers

Figure 13: Forth case (four units operating (1, 3, 4 \&6))

\subsection{Fifth case (four units operating $(2,3,4 \& 5)$}

The results of the streamlines of the fifth scenario after adding routers indicate that, a good distribution at all entrances to the units, as shown in Figure (14-a) and there is improvement in the density of the flow lines at the bottom of the bell mouth and uniform distribution of the incoming streamlines of the working units, as shown in Figure (14-a). While velocity distribution shows a clear positive change for the relatively low velocity zone leading to increasing velocities in this area especially at unit number 6 that it was always affected by this phenomenon, as shown in Figure (14-b), while, from this figures it shows lack effect of the vortices and the low impact of the separation.

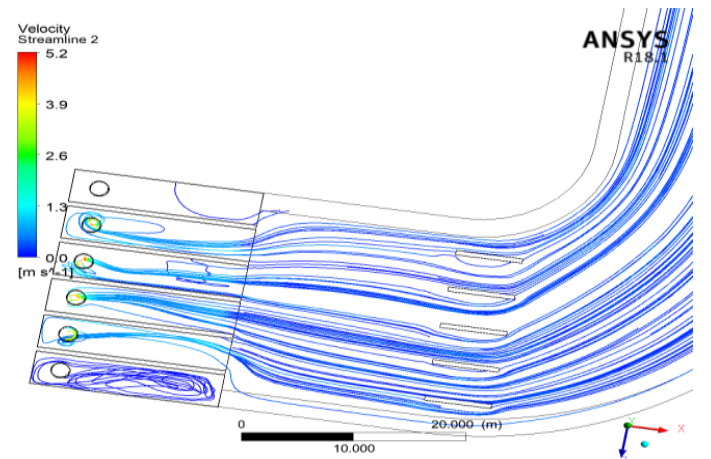

(a) Flow lines at the level of the bell for the fifth scenario after adding routers

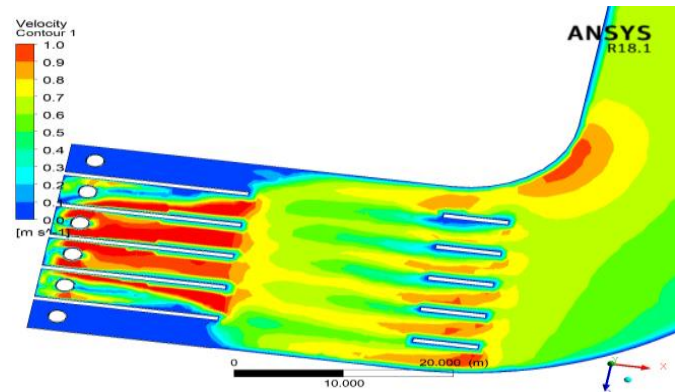

(b) Map of velocities for the fifth scenario after adding routers

Figure 14: Fifth case (four units operating $(2 \& 3 \& 4 \& 6))$

\section{CONCLUSIONS}

In this paper, effects of the curvature suction sump intake of El Tabya pumping station were studied analytically. The paper discussed the installation of routers at the suction sump. From analytical the flow conditions of existing suction sump, there are two problems were illustrated, first problem is the poor distribution for the streamlines of the water at the suction to the units due to a sharp bend in the suction sump that impedes the stability of flow in the entrance of the units, especially when units (5, $6)$ or $(1,2)$ run together at the same time. Where the second problem is the presence of areas where velocities are relatively low so, it become places of accumulation of sediments, which affects the work of the units. The research show that existing sump shape generates swirl flow and appear dead zones in the inner curvature of the intake when operate six units at the same time. The installation of routers with curvature length $6 \mathrm{~m}$ and width $0.5 \mathrm{~m}$ for each pump reduce the pre-swirling surface flow and improves the suction flow condition. 


\section{REFERENCES}

[1] Anwar HO, Weller J, Amphlet MB. 1978. Similarity of free-vortex at horizontal intake. J Hydraul Res. 16:95-105.

[2] Borghei SMKabiri Samani AR. 2003. Critical submergence of vertical intakes using anti-vortex plates. Proceedings of $6^{\text {th }}$ International Conference in Civil Engineering, IUT, Isfahan,Iran, Vol. 7, p. 59-66.

[3] Carriveau R. 2006. The hydraulic vortex - an autocatakinetic system. Int J Gen Syst. 35:707-726.

[4] Constantinescu GS, Patel VC. 1998. Role of Turbulence model in prediction of pump intake vortices. New York: ASME FEDSM98-5003.

[5] Daggett LL, Keulegan GH. 1974. Similitude in free surface vortex formations. J Hydraul Div ASCE. 100:1565-1580.

[6] Dicmas JL. 1987. Vertical turbine, mixed flow and propeller pumps. New York: McGraw-Hill.

[7] Emdad H, Nikseresht AH. 2003. Application of vortex lattice and quasi-vortex lattice method with free wake in calculation of aerodynamic characteristics of a hovering helicopter rotor blade in ground effect. Sci Iran. 10:84-90.

[8] Enomoto T, Tagomori M, Goto A. 1995. Turbomachinery. 23:165-168.

[9] Hannani SK, Parsinejad F. 2003. Incompressible stokes flow calculation using a finite point method. Sci Iran. 10:44-55.

[10] Hite EJ Jr, Mih WC. 1994. Velocity of air-core vortices at hydraulic intakes. JHydraul Eng ASCE. 120:284-297.

[11] Hydraulic Institute. 1998. Centrifugal/Vertical Pump Intake design, appendix D.4 intake design consideration. New York: ANSI/HI. ISBN 1880952-26-2

[12] Jain AK, Ranga Raju KG, Garde RJ. 1978. Vortex formation at vertical pipe intakes. J Hydraulics Div ASCE. 104:1429-1445.

[13] Kabiri-Samani AR, Borghei SM, Saidi MH. 2006. Entrapped air in long water tunnels during transition from pressurized to free surface flow regime. Sci Iran. 13:174-186.

[14] Kamemoto K, Toyokura T, Sano A. 1986. A numerical simulation technique for predicting of flow feature of a pump sump.Proc IAHR Symp Montreal. 2:1-14

[15] Karassik IJ, Messina JP, Cooper P, Heald CC. 2001. Pump handbook. New York: McGraw-Hill.

[16] Knauss J. 1987. Swirling flow problems at intakes. Hydraulic structures design manual 1. Rotterdam: A.A., IAH, Balkema.

[17] Launder BE, Spalding DB. 1974. The numerical computation of turbulent flows. Comput Methods Appl Mech Eng. 3:269-289.

[18] Nagahara T, Sato T, Okamura T. 2003. Measurement of the flow around the submerged vortex cavitation in a pump intake by means of PIV. Fifth International Symposium on Cavitation, Osaka.

[19] Odgaard AJ. 1986. Free-surface air-core vortex. J Hydraul Eng ASCE. 112:610-620.

[20] Padmanabhan M, Hecker GE. 1984. Scale effect in pump sump models. J Hydraul Div ASCE. 110:1540-1556.

[21] Reddy YR, Pickford JA. 1972. Vortices at intake in conventional sumps. Water Power. 22:108-109.

[22] Rocklage-Marliani G, Schmidts M, Ram VIV. 2003. Three dimensional laser-Doppler velocimeter measurements in swirling turbulent pipe flow. Appl Sci Res Springer Neth.70(1-4):43-67.

[23] Standard method for model testing the performance of a pump sump. JSME S 004. 1984. The Japan Society of Mechanical Engineers. Japanese.

[24] B. E. Launder and D. B. Spalding, "The Numerical Computation of Turbulent Flows", Computer Methods in Applied Mechanics and Engineering, Vol. 3, pp. 269-289, 1974. 